\title{
RESPUESTA in vitro DE MICROBULBILLOS de Rhodophiala montana SOMETIDOS A INDUCCIÓN DE POLIPLOIDÍA EN PRESENCIA DE COLCHICINA*
}

\section{In vitro RESPONSE OF MICROBULBS OF Rhodophiala montana SUBJECT TO POLYPLOIDY INDUCTION IN PRESENCENCE OF COLCHICINE}

\author{
Jara, G.', Seemann, P.', Muñoz, M.', Riegel, R. ', Schiappacasse, F. ${ }^{2}$, Peñailillo, P. ${ }^{2}$ y Vico, V.' \\ 'Instituto de Producción y Sanidad Vegetal, Universidad Austral de Chile, Valdivia, hile. \\ E-mail: gjara@uach.cl \\ ${ }^{2}$ Departamento de Horticultura, Universidad de Talca, Talca, Chile.
}

\section{INTRODUCCIÓN}

En floricultura se busca que las plantas cultivadas tengan grandes flores y fuertes tallos. Esto ha llevado al uso preferencial de individuos poliploides como material parental, siempre que éstos se encuentren disponibles en forma silvestre o cultivada, en caso contrario puedan ser inducidos por medios artificiales. Las especies de Rhodophiala presentan varias características favorables, sin embargo el aumentar el tamaño de la flor, intensidad de color, altura, etc., las haría más atractivas para diferentes mercados. Hoy en día la mayor parte de los cultivares comerciales corresponden a individuos tetraploides. La poliploidía se ha utilizado en numerosas especies ornamentales, como por ejemplo en Begonia, híbridos de Chrysanthemum indicum, Freesia, Gladiolus, Iris, Narcissus, híbridos de Pelargonium, Prímula sinensis y P. obconica, Rosa, Tagetes patula y Tulipa sp. En las plantas anteriores se han creado individuos tetraploides, e incluso en algunas de ellas se han creado individuos triploides, pentaploides y hasta hexaploides (Meerov, 2000).

Hoy en día se reconoce la inducción de clones poliploides por medio de colchicina, oryzalina u otro medio en diversas especies, así como también han sido desarrolladas técnicas citológicas que permiten reconocer la dotación cromosómica de diferentes individuos (North, 1979). Además las técnicas de cultivo de tejidos in vitro están suficientemente desarrolladas para una multiplicidad de especies cultivadas, incluidas muchas geófitas. Existe información sobre otras bulbosas, la cual debe ser adaptada para alcanzar las características superiores deseadas, así como también se espera que el manejo aplicado a Hippeastrum y a otras especies de similar morfología en otros países, pueda ser aplicado exitosamente en las cuatro especies en estudio. En base estos antecedentes el objetivo del presente trabajo fue evaluar la capacidad de regeneración y crecimiento en microbulbillos de Rhodophiala montana tratados con una solución de colchicina y determinar posteriormente su ploidía.

\section{MATERIAL Y MÉTODOS}

Microbulbillos de Rhodophiala montana fueron cultivados en medio MS líquido, adicionado con colchicina al $0,05 \%$, durante 4 días en agitación constante. Luego este medio fue desechado y reemplazado por uno fresco sin colchicina, en donde fueron sembrados los microbulbillos realizándoles a la mitad de ellos un corte en cruz en la zona basal y la otra mitad se sembraron sin corte en cruz, para ser incubados durante 60 días. Además para comparar el crecimiento de microbulbillos tratados con colchicina se incubaron microbulbillos sin la aplicación de colchicina, con corte en cruz y sin corte, incubándolos durante 30 días. Cada microbulbillo 
fue sembrado en forma individual, en medio MS solidificado con $0,8 \%$ de agar, e incubados en cámara con fotoperiodo de 16 horas luz, flujo de fotones fotosinteticamente activos de 50

$\mu \mathrm{mol} \mathrm{m} \mathrm{m}^{-2}$ y $23^{\circ} \mathrm{C}$, para realizar las siguientes evaluaciones: Número y longitud de brotes y raíces, número y diámetro de microbulbillos y sobrevivencia (\%).

La comprobación de la duplicación cromosómica se realizó en puntas de raíz, mediante tinción citológica y observación microscópica (Grant et. al. 1984).

\section{RESULTADOS Y DISCUSIÓN}

Se observó que el coeficiente de multiplicación fue mayor en los microbulbillos con corte basal, tanto en los testigos y en los tratados con colchicina, presentándose en los microbulbillos trata- dos con colchicina sin corte y con corte en cruz, una menor tasa de sobrevivencia. Con respecto a la duplicación cromosómica se encuentran en evaluación los diferentes genotipos tratados con colchicina, sin embargo se ha logrado producir duplicación cromosómica en algunos genotipos, los cuales se encuentran en multiplicación.

\section{BIBLIOGRAFÍA}

GRANT, J., BROWN, A. Y GRACE, J. 1984. Cytological and Isozyme Diversity in Glicine tomentella Hayata (Leguminosae). Australian Journal of Botan. 32: 665-677.

MEEROW, A. W. 2000. Breeding Amaryllis. In: Callaway, D.J.; Callaway, M.B. (eds.). Breeding ornamental plants. Portland, Timber Press, Inc. pp 174-195.

NORTH, C. 1979. Plant breeding and genetics in horticulture. Londres, The Macmillan Press. 100p.

Cuadro 1 Sobrevivencia y regeneración de $R$. montana sin y con aplicación de colchicina.

Table 1. Survival and regeneration of $R$. montana without and with application of colchicine.

\begin{tabular}{|c|c|c|c|c|c|}
\hline $\begin{array}{l}\text { Evaluaciones in vitro ( } 30 \text { días de } \\
\text { cultivo) }\end{array}$ & \multicolumn{2}{|c|}{$\begin{array}{l}\text { R. montana sin } \\
\text { aplicación de } \\
\text { colchicina } \\
\text { Sin corte Con corte }\end{array}$} & \multirow{2}{*}{$\begin{array}{l}\text { Evaluaciones in vitro (60 días de } \\
\text { cultivo) } \\
\text { Sobrevivencia }(\%)\end{array}$} & \multicolumn{2}{|c|}{$\begin{array}{l}\text { R. montana con } \\
\text { aplicación de } \\
\text { colchicina } \\
\text { Sin corte Con corte }\end{array}$} \\
\hline obrevivencia (\%) & 100 & 100 & & $80,0 \mathrm{a}$ & $40,0 \mathrm{~b}$ \\
\hline úmero de brotes & $1,1 \mathrm{~b}$ & $2,0 \mathrm{a}$ & & & \\
\hline ongitud de brotes $(\mathrm{cm})$ & 4,3 & 4,4 & Longitud de brotes $(\mathrm{cm})$ & & 0,8 \\
\hline Número de raíces & 1,0 & 1,1 & Número de raíces & 0,7 & 0,5 \\
\hline Longitud de raíces $(\mathrm{cm})$ & 2,3 & 1,1 & Longitud de raíces $(\mathrm{cm})$ & 1,5 & 1,1 \\
\hline Número de microbulbillos & $1,0 \mathrm{~b}$ & $2,0 \mathrm{a}$ & Número de microbulbillos & $1,0 \mathrm{~b}$ & $1,6 a$ \\
\hline Diámetro de microbulbillos $(\mathrm{cm})$ & 0,7 & 0,5 & Diámetro de microbulbillos $(\mathrm{cm})$ & $0,4 \mathrm{a}$ & $0,2 b$ \\
\hline
\end{tabular}

*Trabajo Financiado mediante Proyecto FIA-BIOT-01-A-071

Agro Sur $34(1-2): 68-702006$

\title{
GERMINACIÓN DE SEMILLAS DE Rhodophiala spp. SEED GERMINATION OF Rhodophiala spp.
}

\author{
Seemann, P.', Muñoz, M.', Riegel, R.', Jara, G.', Schiapacasse, F.', Peñailillo, P. ${ }^{2}$ y Vico, V. \\ 'Universidad Austral de Chile. Casilla 567 Valdivia, Chile. \\ E-mail: pseemann@uach.cl
}

\section{INTRODUCCIÓN}

Las especies del género Rhodophiala se caracterizan por poseer bulbos tunicados y hojas linear lanceoladas. Sus flores de color amarillo, rosado o rojo de color amarillos, rosado o rojo dispuestas en umbelas, el perigonio en forma de embudo está formado por 6 tépalos. El androceo presenta seis estambres con filamentos rojos y anteras amarillas. Gineceo sincárpico compues- 